\title{
Maternal Antenatal Treatments Influence Initial Oral Microbial Acquisition in Preterm Infants
}

\author{
Karen D. Hendricks-Muñoz, MD, MPH ${ }^{1} \quad$ Guillermo Perez-Perez, $\mathrm{DSc}^{2}$ jie Xu, $\mathrm{PhD}^{1}$ Yang Kim, $\mathrm{MD}^{3}$ \\ Moi Louie, MD ${ }^{3}$ \\ ${ }^{1}$ Division of Neonatal Medicine, Department of Pediatrics, Virginia \\ Commonwealth University School of Medicine, Richmond, Virginia \\ 2 Department of Medicine and Microbiology, New York University \\ School of Medicine, New York, New York \\ ${ }^{3}$ Division of Neonatology, Department of Pediatrics, New York \\ University School of Medicine, New York, New York \\ Address for correspondence and reprint requests Karen D. Hendricks- \\ Muñoz, MD, MPH, Professor of Pediatrics; Chief, Division of Neonatal \\ Medicine, Department of Pediatrics, Virginia Commonwealth \\ University Medical Center, 1001 E. Marshall St, Richmond, VA 23228 \\ (e-mail: hendrk01@gmail.com).
}

Am J Perinatol 2013;30:47-52.

\begin{abstract}
Keywords

- antenatal therapy

- preterm infant

- oral microbiota

- Lactobacillus

Objective The purpose of this study was to analyze the association of maternal antenatal therapy on initial preterm infant oral microbial acquisition of gut metabolically important bacteria: Firmicutes, Bacteroidetes, Lactobacillus, Bifidobacterium, and Bacteroides species.

Study Design Infant oral samples were collected prefeeding at 24 hours and analyzed using group-specific primers by real-time 165 rRNA quantitative polymerase chain reaction with analysis of variance and logistic regression to evaluate effect of antenatal exposure.

Results Sixty-five infants $<34$ weeks' gestational age (GA) were evaluated; mean GA was $28.6 \pm 2.6$ (standard deviation) weeks. Infants unexposed to antenatal treatment $(n=5)$ acquired $<1 \%$ Firmicutes, which was composed of $100 \%$ Lactobacillus species with no detectable Bifidobacterium, Bacteroidetes, or Bacteroides species. Infants exposed to antibiotics $(n=7)$, acquired fivefold less total bacterial density (TBD) with $45 \%$ Firmicutes 1.3\% Lactobacillus species, 23.5\% Bacteroidetes and rare Bacteroides. Compared with unexposed infants, steroids $(n=26)$ or steroid and antibiotics $(n=27)$ exposure led to an eightfold increase in TBD with $<1 \%$ Lactobacillus species and Bacteroides species $100 \%$ and $30 \%$, respectively $(p<0.04)$. Bifidobacterium was undetectable in all groups.

Conclusion Preterm infant exposure to routine maternal antenatal treatments influence early oral microbial acquisition during the primary hours related to establishment of gut commensal bacteria.
\end{abstract}

In the naïve edentulous preterm infant, oral microbiota influence the initial pattern of bacteria exposure available for establishment of gut bacterial colonization. ${ }^{1-3}$ Despite their importance, factors that influence the patterns of these early oral bacterial colonizers have not been described. In the neonatal intensive care unit (NICU), preterm infants are indirectly exposed to antibiotics and steroids through ante- natal maternal treatments. Recently, antibiotic treatment begun at birth and continued treatment have been associated with increased risk of necrotizing enterocolitis (NEC), implicating that initial establishment of microbial repertoires may be important for early mucosal protective properties or injury. ${ }^{4}$ Furthermore, oral early acquired commensal bacterial colonization patterns, altered by cesarean delivery, have received

December 11, 2011

accepted after revision

March 15, 2012

published online

July 13, 2012
Copyright $\odot 2013$ by Thieme Medical Publishers, Inc., 333 Seventh Avenue, New York, NY 10001, USA. Tel: +1(212) 584-4662.
DOI http://dx.doi.org/ 10.1055/s-0032-1321499. ISSN $0735-1631$. 
been linked to later infant health and development of caries. ${ }^{5-7}$

In most clinical settings, it is common to treat women who present with risk for preterm delivery with antenatal steroids to accelerate fetal lung maturity and antenatal antibiotics to protect the preterm infant from infection risk. ${ }^{8-11}$ Although antenatal treatments are beneficial for infant health, they may indirectly influence the initial preterm oral bacterial acquisition patterns important for later gut microbe colonization. For these reasons, we examined the impact of maternal antenatal steroid and antibiotic treatment on preterm infant initial oral bacterial acquisition patterns to identify preliminary microbiota repertoire patterns as a basis to study the impact of early acquired bacterial patterns on subsequent preterm infant health.

\section{Material and Methods}

\section{Patient Recruitment}

The study was approved by the Human Research Review Board of New York University School of Medicine and Bellevue Hospital Center. Mothers who delivered a preterm infant at less than 34 weeks' gestation signed informed written consent as required by the Institutional Review Board for their infant's participation.

\section{Patient Sampling}

Infant samples were obtained at 24 hours of life using a sterile dry soft swab that was rolled along the infant's oral mucosal surface of the mouth, inner cheeks, and tongue until saturated with saliva, placed in $2.0 \mathrm{~mL}$ of phosphate-buffered saline, centrifuged at 14,000 rpm for 6 minutes, and the pellets stored at $-80^{\circ} \mathrm{C}$ prior to processing. Samples were analyzed by the molecular methods described later to identify bacterial
DNA. All infants included were treated with antibiotics within 30 minutes of admission to the NICU consisting of ampicillin and gentamicin, and no infant received any feedings prior to oral sampling. Those mothers treated with antenatal steroids received a complete 48-hour treatment of betamethasone or dexamethasone. Antibiotics, when provided, consisted of ampicillin or erythromycin. One mother received ampicillin and gentamicin and one mother received ampicillin and azithromycin. In our center, chorioamnionitis was defined as maternal fever $\left(>37.8^{\circ} \mathrm{C}\right)$ associated with two or more of the following: maternal tachycardia $>100$ beats/min, fetal tachycardia $>160$ beats $/ \mathrm{min}$, maternal serum leukocytosis $>15,000 / \mathrm{mm}^{3}$, uterine tenderness, or malodorous vaginal discharge. Infant demographic data such as birth weight, gestational age, and race as well as maternal medical diagnoses and medical treatments were collected.

\section{Bacterial DNA Preparation}

Bacterial DNA was isolated with MasterPure DNA Purification Kit (EPICENTRE Biotechnologies, Madison, WI), as described by manufacturer. DNA was stored at $-20^{\circ} \mathrm{C}$ until analysis.

\section{5 rRNA Polymerase Chain Reaction}

The bacterial specific primers used are listed in - Table $\mathbf{1}$. Oligo nucleotide primers and the Power SYBR Green PCR Master Mix were purchased from Applied Biosystems (Carlsbad, CA) based on previous Firmicutes and Bacteroidetes phyla and Bifidobacteria, Lactobacillus, and Bacteroides species detection analysis. ${ }^{12-16}$ Detection of DNA polymerase chain reaction (PCR) was performed with the 7900HT Fast Real-Time PCR System (Applied Biosystems) using optical grade 384-well plates with control standards determined by automatic analysis settings. Duplicate samples were used for the determination of DNA by real-time

Table 1 Primer List for Targeted Bacterial Analysis

\begin{tabular}{|c|c|c|c|}
\hline Target Organisms & Primer & Sequence $\left(5^{\prime}\right.$ to $\left.3^{\prime}\right)$ & $\begin{array}{l}\text { Annealing } \\
\text { Temp }\left({ }^{\circ} \mathrm{C}\right)\end{array}$ \\
\hline \multirow[t]{2}{*}{ Bacteroidetes $^{12}$ (126 bp) } & Forward primer & GGARCATGTGGTTTAATTCGATGAT & 60 \\
\hline & Reverse primer & AGCTGACGACAACCATGCAG & \\
\hline \multirow[t]{2}{*}{ Firmicutes $^{12}$ (126 bp) } & Forward primer & GGAGYATGTGGTTTAATTCGAAGCA & 60 \\
\hline & Reverse primer & AGCTGACGACAACCATGCAC & \\
\hline \multirow[t]{2}{*}{ All bacteria $^{13}(200 \mathrm{bp})$} & Forward primer & ACTCCTACGGGAGGCAGCAG & 60 \\
\hline & Reverse primer & ATTACCGCGGCTGCTGG & \\
\hline \multirow[t]{2}{*}{ Lactobacillus $^{14}$ (90 bp) } & Forward primer & TACATYCCAACHCCAGAACG & 60 \\
\hline & Reverse primer & AAGCAACAGTACCACGACCA & \\
\hline Lac-Probe & & (FAM)AAGCCATTCTTRATGCCAGTTGAA(TAMRA) & \\
\hline \multirow[t]{2}{*}{ Bifidobacterium $^{15}$ (553 bp) } & Forward primer & CTCCTGGAAACGGGTGG & 55 \\
\hline & Reverse primer & GGTGTTCTTCCCGATATCTACA & \\
\hline \multirow[t]{2}{*}{ Bacteroides $^{16}$ (106 bp) } & Forward primer & GAGAGGAAGGTCCCCCAC & 60 \\
\hline & Reverse primer & CGCTACTTGGCTGGTTCAG & \\
\hline AllBac-probe & & (FAM)CCATTGACCAATATTCCTCACTGCTGCCT(TAMRA) & \\
\hline
\end{tabular}


PCR, and mean values were calculated. The bacteria density for each target group was calculated using standard curves generated by $16 \mathrm{~S}$ rRNA sequence containing plasmids. ${ }^{12}$ These primers amplify $90 \%$ of the rRNA coding sequence and minimize PCR bias. The PCR reaction was performed in a total volume of $10 \mu \mathrm{L}$. Bacteroidetes, Firmicutes, and total bacterial densities were detected using $100 \mathrm{nmol}$ each of the forward and reverse primers and $1 \mathrm{ng}$ of DNA for each reaction. PCR conditions for amplification were $50^{\circ} \mathrm{C}$ for 2 minutes, $95^{\circ} \mathrm{C}$ for 10 minutes, and 40 cycles of $95^{\circ} \mathrm{C}$ for 15 seconds and $60^{\circ} \mathrm{C}$ for 1 minute. A melting curve analysis was done after amplification. ${ }^{13-16}$

\section{Statistical Methods}

Total bacterial density and specific bacteria phyla as well as the selected species in saliva were compared between exposed and nonexposed samples. Infants were grouped into four groups: no exposure, antibiotics, steroids, and steroids and antibiotics groups. Data were analyzed (SPSS 16 for Windows, SPSS, Inc., Chicago, IL) using descriptive, parametric, and nonparametric statistics according to the level of data obtained and the examination of the assumptions underlying the tests. All values were expressed as mean \pm standard error of the mean unless otherwise indicated. Univariate analyses were performed to assess the distribution and variability of the data and to describe the sample. One-way analysis of variance and $t$ test were used to assess differences in the quantity (density) of each bacterial phyla or species pattern within the defined groups. With adjustments for potential confounding factors, separate multiple logistic regression analyses were performed to assess impact of gender, race, and mode of delivery on microbial density and patterns. Statistical significant difference was defined as $p<0.05$.

\section{Results}

\section{Baseline Characteristics}

Sixty-five preterm newborns born at $<34$ weeks' gestation participated in this study. Demographic characteristics of the total population are described in - Table 2. Five (7.6\%) infants were not exposed to any medication, 7 (10.7\%) infants were exposed to antibiotics only, 26 infants (40\%) were exposed to only antenatal steroids, and 27 (41.5\%) were exposed to both steroids and antibiotics. Of those mothers who received antibiotics, all demonstrated maternal fever $>37.8^{\circ} \mathrm{C}$ associated with maternal tachycardia $>100$ beats/min and fetal tachycardia $>160$ beats/min. No mother received antibiotics after birth. Fifty-three infants (81.5\%) were delivered by cesarean section and $12(18.5 \%)$ by vaginal delivery. No infant was diagnosed with early onset bacteremia diagnosed as a positive blood culture within the first week of life. Overall mean gestational age ( \pm standard deviation) for the group was $28.6 \pm 2.6$ weeks with mean birth weight $1176 \pm 357 \mathrm{~g}$. Thirty-two infants were singletons and 23 were multiples. Thirty-four were males and 31 were females of a variety of racial and ethnic backgrounds ( - Table 2 ). Regression analyses did not show any likelihood of a microbial density or pattern significantly altered with infant mode of delivery ethnicity or infant gender.

Table 2 Maternal Characteristics $(n=65)$

\begin{tabular}{|c|c|c|c|c|}
\hline Characteristics & $\begin{array}{l}\text { No Medications } \\
(n=5)\end{array}$ & $\begin{array}{l}\text { Steroids } \\
(n=26)\end{array}$ & $\begin{array}{l}\text { Steroids and } \\
\text { Antibiotics }(n=27)\end{array}$ & $\begin{array}{l}\text { Antibiotics } \\
(n=7)\end{array}$ \\
\hline \multicolumn{5}{|l|}{ Diagnosis } \\
\hline PPROM & 0 & 9 & 6 & 4 \\
\hline Hypertension/preeclampsia & 3 & 8 & 8 & 0 \\
\hline Incompetent cervix/NRFHT & 1 & 4 & 5 & 2 \\
\hline Chorioamnionitis & 0 & 0 & 5 & 2 \\
\hline Previa with bleeding & 1 & 5 & 3 & 0 \\
\hline Cesarean birth $(n=53), n(\%)$ & $4(80)$ & $24(92)$ & $20(74)$ & $5(71)$ \\
\hline Multiples $(n=23), n(\%)$ & $2(40)$ & $7(27)$ & $11(41)$ & $3(43)$ \\
\hline Males $(n=34), n(\%)$ & $4(80)$ & $16(61.5)$ & $9(33)^{*}$ & $5(71)$ \\
\hline \multicolumn{5}{|l|}{ Race/Ethnicity, n (\%) } \\
\hline Non-Hispanic white $(n=18)$ & $0(0)$ & $7(27)$ & $10(37)$ & $1(14)$ \\
\hline Hispanic $(n=11)$ & $2(40)$ & $3(12)$ & $6(22)$ & $0(0)$ \\
\hline Asian $(n=15)$ & $1(20)$ & $9(35)$ & $4(15)$ & $1(14)$ \\
\hline Non-Hispanic black $(n=21)$ & $2(40)$ & $7(27)$ & $7(26)$ & $5(71)$ \\
\hline
\end{tabular}

PPROM, preterm premature rupture of membranes; NRFHT, nonreactive fetal heart tracing.

One-way ANOVA T- test.

"Statistically significant $(p<0.05)$ from no medications group.

$\mathrm{N}$ represents the number of samples in each group \pm SE (Standard Error). 
Table 3 Infant Oral Microflora in Relation to Maternal Antenatal Treatment

\begin{tabular}{|c|c|c|c|c|}
\hline Bacterial DNA/mL & $\begin{array}{l}\text { No Medications } \\
(n=5)\end{array}$ & Steroids $(n=26)$ & $\begin{array}{l}\text { Steroids and Antibiotics } \\
(n=27)\end{array}$ & $\begin{array}{l}\text { Antibiotics } \\
(n=7)\end{array}$ \\
\hline \multicolumn{5}{|c|}{ Category: Mean $( \pm \mathrm{SE}) \times 10^{5}$} \\
\hline Firmicutes & $0.145 \pm 0.06$ & $249.5 \pm 41.1^{\mathrm{a}}$ & $96.6 \pm 36.3^{\mathrm{a}}$ & $3.2 \pm 1.8^{\mathrm{a}}$ \\
\hline$\%$ Firmicutes $^{\mathbf{b}}$ & 0.4 & 88.0 & 76.4 & 45.0 \\
\hline \% Lactobacillus ${ }^{\mathrm{c}}$ & 100 & 0.05 & 0.16 & 1.3 \\
\hline Fold $\Delta \%$ Firmicutes & & +220.0 & +191.0 & +112.5 \\
\hline Bacteroidetes & $0.007 \pm 0.0$ & $0.034 \pm 0.0^{\mathrm{a}}$ & $1.96 \pm 0.00^{\mathrm{a}}$ & $1.67 \pm 0.06^{\mathrm{a}}$ \\
\hline \% Bacteroidetes ${ }^{\mathbf{b}}$ & 0.2 & 0.1 & 1.6 & 23.6 \\
\hline$\%$ Bacteroides $^{\mathrm{d}}$ & 00.00 & $100 \%$ & $30.3 \%$ & $0.01 \%$ \\
\hline Fold $\Delta \%$ Bacteroidetes & & -2.0 & +8.0 & +118.0 \\
\hline Total bacteria density & $35.7 \pm 10.4$ & $283.4 \pm 71.5^{a}$ & $126.3 \pm 55.3^{a}$ & $7.09 \pm 2.38^{\mathrm{a}}$ \\
\hline Fold $\Delta$ total density & & +7.9 & +3.5 & -5.0 \\
\hline$\%$ other bacteria $^{\mathrm{e}}$ & 99.4 & 11.9 & 22.0 & 31.4 \\
\hline
\end{tabular}

One-way analysis of variance $t$ test. $n$, number of samples in each group $\pm \mathrm{SE} ; \Delta$, fold change; SE, standard error.

${ }^{a}$ Statistically significant $(p<0.05)$ compared with no medications group.

bercent of total bacteria density.

'Percent of Lactobacillus of Firmicutes density.

${ }^{\mathrm{d}}$ Percent of Bacteroides of Bacteroidetes density.

ePercent of total density that were other bacterial phylum.

\section{Oral Flora Characteristics without Antenatal Medications}

Bacterial counts for the unexposed infant group are summarized in - Table 3 . In untreated infants, the Firmicutes metabolic phylum, although not prominent at $<1 \%$ of the total bacterial density, was composed completely of Lactobacillus species (-Figs. 1 and 2). Organisms of the Bacteroidetes phylum were barely detectable, and species from Bifidobacterium or Bacteroides were absent.

Oral Flora Characteristics with Antenatal Steroids and Antenatal Steroids and Antibiotics

Total bacterial density was almost eightfold greater in infants exposed to antenatal steroids compared with the unexposed group with an increased composition of Firmicutes (88.0\%) metabolic repertoire during this early period (-Fig. 1, - Table 3). Despite the greater density in Firmicutes, Lactobacillus prevalence was decreased to $0.05 \%$ (-Fig. 2, - Table 3). Density levels of the Bacteroidetes were significantly increased by 10 -fold compared with unexposed infant levels composed completely of Bacteroides species (-Fig. 3, - Table 3). The addition of antibiotics with antenatal steroids also resulted in a 3.6-fold increase in total bacterial density compared with the unexposed group and an almost 1000-fold increase in Firmicutes density (-Fig. 1, - Table 3). Additionally, the prevalence of Lactobacillus species was minimal, decreased to $0.16 \%$ (-Fig. 2, - Table 3). Density levels of the Bacteroidetes

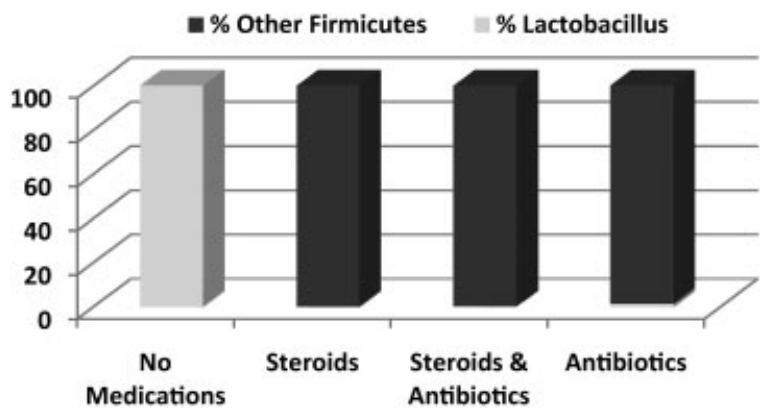

Figure 2 Antenatal treatment effects on infant oral Lactobacillus and other Firmicutes species. The percent of Lactobacillus and other Firmicutes species in proportion to the total Firmicutes bacterial density in infant oral microflora. Total number of infants $=65$, no medications $(n=5)$, steroid $(n=26)$, steroid and antibiotics $(n=27)$, antibiotics $(n=7)$. 


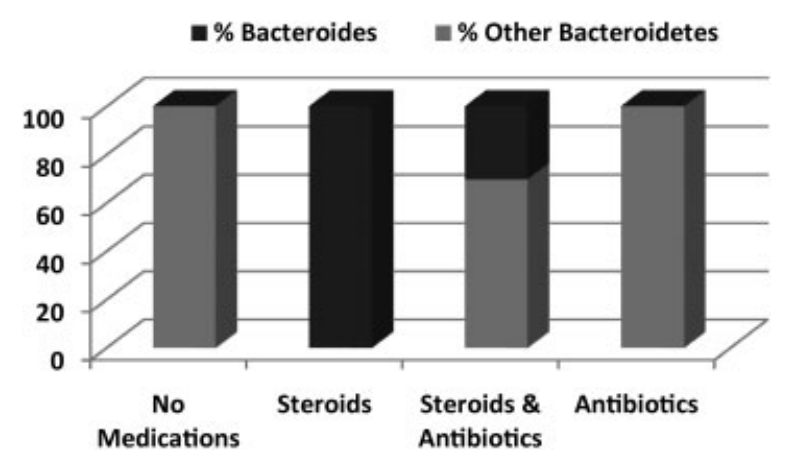

Figure 3 Antenatal treatment effects on infant oral Bacteroides species and other Bacteroidetes species. The percent of Bacteroides and other Bacteroidetes species in proportion to the total Bacteroidetes bacterial density in infant oral microflora. Total number of infants $=65$, no medications $(n=5)$, steroid $(n=26)$, steroid and antibiotics $(n=27)$, antibiotics $(n=7)$.

increased almost 300-fold to the greatest levels of all groups reviewed. In this group, 30.3\% of Bacteroidetes were of the Bacteroides species ( - Fig. 3, - Table 3). Of the total bacterial density in this group, $76.4 \%$ were of the metabolic repertoire (-Fig. 1, - Table 3). The Firmicutes to Bacteroidetes ratio was notably increased to 49.3 .

\section{Oral Flora Characteristics of Antenatal Antibiotics}

Infants exposed only to antenatal antibiotics demonstrated a suppressed total bacterial density fivefold lower than the unexposed group but a pattern of 20 -fold increase in Firmicutes density (-Fig. 1, - Table 3). The prevalence of Lactobacillus species was depressed to $1.3 \%$. Density levels of the Bacteroidetes increased by almost 250-fold compared with unexposed infant levels $(p<0.02)$, but Bacteroides species

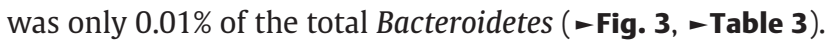

\section{Discussion}

The preterm infant's oral cavity provides a gateway for first mucosal immune and gut colonizers for later overall intestinal function and nutritional health. ${ }^{17-20}$ Disruption of early colonizers can impact on later bacterial acquisition, such as occurs during cesarean delivery where colonization changes have been associated with later intestinal patterns and specific long-term health risks of infant caries. ${ }^{5,6}$ Recently, early antibiotic exposure in preterm infants has been associated with an increased risk of later NEC. ${ }^{4}$ Additionally, specific health-promoting bacteria, Lactobacillus and Bifidobacterium, provided after birth to the preterm infant appear to be important therapies that have been associated with decreased risk of later NEC development. ${ }^{21}$ Furthermore, the use of bacteria in therapeutic maternal antenatal probiotics has been associated with a decrease risk in later childhood atopic disease. $^{22,23}$ These investigations support the important role of the establishment of bacterial colonization patterns in infant health and disease. Using quantitative 16S PCR technology, we provide additional data that demonstrate that routinely provided antenatal maternal treatment are also indirect mechanisms that alter preterm infant initial bacterial patterns and total bacterial density acquisition levels. Our infants were predominantly delivered by cesarean birth, and exposure to maternal antenatal common treatments influenced early preterm oral microbial acquisition.

Our results also detail patterns of specific bacteria during this acquisition period-specifically, that Firmicutes, Bacteroidetes, and Bifidobacterium of the Actinobacteria phyla oral acquisition is initially extremely limited in medication unexposed infants. Despite the small numbers, the results consistently demonstrate that the initial bacterial acquisition pattern is relatively devoid of these organisms. However, those Firmicutes present were completely of the Lactobacillus species. Furthermore, despite its prevalence in vaginal fluid, in all of our population samples Bifidobacterium was absent.

In contrast, infants exposed to any antenatal steroid treatments with or without antibiotics facilitated more oral bacterial density with a pattern composed primarily of Firmicutes with little Lactobacillus species but a greater prevalence of Bacteroides species. As expected, antenatal antibiotics suppressed preterm infant total bacterial acquisition but unexpectedly altered the acquisition pattern, favoring an increase in Bacteroidetes density to almost a quarter (23.5\%) of the total bacterial density. Additionally, antibiotics limited Bacteroides species to $<1 \%$. Firmicutes density was increased compared with untreated infants with little Lactobacillus species. In these antibiotic-only infants the contribution of other phylum was decreased to $31 \%$ of the total oral density.

The use and efficacy of antenatal steroids has been well established for preterm infant lung maturation.8,24,25 Additionally, steroids have additional benefits for infant survival including prevention of NEC. ${ }^{26}$ Further understanding of the impact of antenatal exposure in the full-term infant needs to be explored as our investigations were limited to the preterm infant and the depth of impact of antenatal treatment on acquisition of early commensal microbiota may be varied in the immature infant. It is known that antenatal amoxicillin for group B streptococcal prophylaxis in the term infant is associated with decreased Clostridium stool colonization at 3 days of life compared with untreated infants using culture-dependent methods. ${ }^{27}$ In our study, the small numbers of unexposed infants are a limitation in our observations; however, our current obstetric practice is to provide antenatal steroids to these high-risk infants. Those infants whose mothers received antenatal antibiotics did so due to perceived infection risk to the infant. Nevertheless, the bacterial density and acquisition patterns even in those infants whose mothers were treated for suspected chorioamnionitis or PPROM were strikingly similar within groups, providing support that maternal antenatal treatments indirectly impact on infant early microbial acquisition. Thus, the results of our study support an influence of altered bacterial acquisition patterns in those infants exposed to antenatal steroid with or without antibiotics. Furthermore, when used alone, antenatal antibiotics suppressed oral colonization density and bacterial diversity in support of previous studies that describe decreased microbial diversity in infants exposed to early antibiotics. ${ }^{28,29}$

Our results outline alteration of unique oral bacterial acquisition patterns acquired in preterm infants exposed to 
maternal antenatal steroids and antibiotics. Given the importance of the oral cavity in the acquisition of early gut microbes in the infant, our results further validate that maternal treatments alter infant bacterial acquisition, supporting antenatal therapy as an avenue of indirect infant therapy. Furthermore, our results identify the potential value of saliva as well as oral bacterial acquisition patterns as possible future biomarkers in the preterm infant. Finally, the results offer an initial step in investigating further potential mucosal changes that are important in the selection of specific host microbes to provide opportunities to follow bacterial and mucosal immune patterns to enhance our understanding of the role of early commensal bacteria acquisition important for immune and gut health during a pivotal period in these vulnerable preterm infants.

\section{Acknowledgments}

This work was performed at New York University Langone Medical Center, Bellevue Hospital Center, and Metropolitan Hospital Center. The authors thank Sukuma Narasimhulu, M.D., and Ricardo Mora, M.D., for assistance with patient enrollment and sample collection. The project described was supported by Award Number R21HD059047 from the Eunice Kennedy Shriver National Institute of Child Health and Human Development. The content is solely the responsibility of the authors and does not necessarily represent the official views of the Eunice Kennedy Shriver National Institute of Child Health and Human Development or the National Institutes of Health. This work was partly supported by an educational research grant to K.D. HendricksMuñoz from the Jack Cary Eichenbaum Foundation.

\section{References}

1 Palmer C, Bik EM, DiGiulio DB, Relman DA, Brown PO. Development of the human infant intestinal microbiota. PLoS Biol 2007;5: e177

2 Mackie RI, Sghir A, Gaskins HR. Developmental microbial ecology of the neonatal gastrointestinal tract. Am J Clin Nutr 1999;69: 1035S-1045S

3 Fanaro S, Chierici R, Guerrini P, Vigi V. Intestinal microflora in early infancy: composition and development. Acta Paediatr Suppl 2003;91:48-55

4 Alexander VN, Northrup V, Bizzarro MJ. Antibiotic exposure in the newborn intensive care unit and the risk of necrotizing enterocolitis. J Pediatr 2011;159:392-397

5 Grönlund MM, Lehtonen OP, Eerola E, Kero P. Fecal microflora in healthy infants born by different methods of delivery: permanent changes in intestinal flora after cesarean delivery. J Pediatr Gastroenterol Nutr 1999;28:19-25

6 Li Y, Caufield PW, Dasanayake AP, Wiener HW, Vermund SH. Mode of delivery and other maternal factors influence the acquisition of Streptococcus mutans in infants. J Dent Res 2005;84:806-811

7 Kalliomäki M, Kirjavainen P, Eerola E, Kero P, Salminen S, Isolauri E. Distinct patterns of neonatal gut microflora in infants in whom atopy was and was not developing. J Allergy Clin Immunol 2001; 107:129-134

8 Roberts D, Dalziel S. Antenatal corticosteroids for accelerating fetal lung maturation for women at risk of preterm birth (review). Coch Collab 2006;(4):1-141
9 Ward RM. Pharmacologic enhancement of fetal lung maturation. Clin Perinatol 1994;21:523-542

10 Lee BH, Stoll BJ, McDonald SA, Higgins RD; National Institute of Child Health and Human Development Neonatal Research Network. Adverse neonatal outcomes associated with antenatal dexamethasone versus antenatal betamethasone. Pediatrics 2006;117: 1503-1510

11 American College of Obstetricians and Gynecologists Committee on Obstetric Practice. ACOG Committee Opinion No. 402: Antenatal corticosteroid therapy for fetal maturation. Obstet Gynecol 2008;111:805-807

12 Guo X, Xia X, Tang R, Zhou J, Zhao H, Wang K. Development of a real-time PCR method for Firmicutes and Bacteroidetes in faeces and its application to quantify intestinal population of obese and lean pigs. Lett Appl Microbiol 2008;47:367-373

13 Fierer N, Jackson JA, Vilgalys R, Jackson RB. Assessment of soil microbial community structure by use of taxon-specific quantitative PCR assays. Appl Environ Microbiol 2005;71:4117-4120

14 Armougom F, Henry M, Vialettes B, Raccah D, Raoult D. Monitoring bacterial community of human gut microbiota reveals an increase in Lactobacillus in obese patients and Methanogens in anorexic patients. PLoS ONE 2009;4:e7125

15 Matsuki T, Watanabe K, Fujimoto J, et al. Quantitative PCR with $16 \mathrm{~S}$ rRNA-gene-targeted species-specific primers for analysis of human intestinal bifidobacteria. Appl Environ Microbiol 2004; 70:167-173

16 Guo X, Xia X, Tang R, Wang K. Real-time PCR quantification of the predominant bacterial divisions in the distal gut of Meishan and Landrace pigs. Anaerobe 2008;14:224-228

17 Rook GA, Stanford JL. Give us this day our daily germs. Immunol Today 1998;19:113-116

18 Stappenbeck TS, Hooper LV, Gordon JI. Developmental regulation of intestinal angiogenesis by indigenous microbes via Paneth cells. Proc Natl Acad Sci U S A 2002;99:15451-15455

19 Thompson FM, Catto-Smith AG, Moore D, Davidson G, Cummins AG. Epithelial growth of the small intestine in human infants. J Pediatr Gastroenterol Nutr 1998;26:506-512

$20 \mathrm{Xu} \mathrm{J}$, Gordon JI. Honor thy symbionts. Proc Natl Acad Sci U S A 2003;100:10452-10459

21 Lin HC, Su BH, Chen AC, et al. Oral probiotics reduce the incidence and severity of necrotizing enterocolitis in very low birth weight infants. Pediatrics 2005;115:1-4

22 Kalliomäki M, Salminen S, Arvilommi H, Kero P, Koskinen $\mathrm{P}$ Isolauri E. Probiotics in primary prevention of atopic disease: a randomised placebo-controlled trial. Lancet 2001;357:1076-1079

23 Dotterud CK, Storrø O, Johnsen R, Øien T. Probiotics in pregnant women to prevent allergic disease: a randomized, double-blind trial. Br J Dermatol 2010;163:616-623

24 Liggins GC, Howie RN. A controlled trial of antepartum glucocorticoid treatment for prevention of the respiratory distress syndrome in premature infants. Pediatrics 1972;50:515-525

25 Crowley P, Chalmers I, Keirse MJ. The effects of corticosteroid administration before preterm delivery: an overview of the evidence from controlled trials. Br J Obstet Gynaecol 1990; 97:11-25

26 Bauer CR, Morrison JC, Poole WK, et al. A decreased incidence of necrotizing enterocolitis after prenatal glucocorticoid therapy. Pediatrics 1984;73:682-688

27 Jauréguy F, Carton M, Panel P, Foucaud P, Butel M-J, DoucetPopulaire F. Effects of intrapartum penicillin prophylaxis on intestinal bacterial colonization in infants. J Clin Microbiol 2004; 42:5184-5188

28 Sullivan A, Edlund C, Nord CE. Effect of antimicrobial agents on the ecological balance of human microflora. Lancet Infect Dis 2001;1:101-114

29 Jernberg C, Löfmark S, Edlund C, Jansson JK. Long-term ecological impacts of antibiotic administration on the human intestinal microbiota. ISME J 2007;1:56-66 